\title{
Adaptive Art - A Shape Language Driven Approach to Communicate Dramaturgy and Mood
}

\author{
René Bühling ${ }^{1}$, Emilie Brihi ${ }^{2}$, Michael Wißner ${ }^{1}$, and Elisabeth André ${ }^{1}$ \\ 1 Augsburg University, Human Centered Multimedia, \\ Institute of Computer Science, \\ Universitätsstr. 6a, 86159 Augsburg, Germany \\ \{buehling, wissner, andre\}@informatik.uni-augsburg.de \\ 2 McGill University, \\ Montreal, Quebec H3A 2T5, Canada \\ emilie.brihi@mail.mcgill.ca
}

\begin{abstract}
Graphic designers and visual artists express their narrative intentions using visual tools which essentially include the styling of shapes. In classic art forms design decisions are limited to the initial creation process. Although technical progress makes it possible to adjust contentwise dramaturgy in real-time, the graphical design process often still has static characteristics. In our current work we therefore study how dramaturgical non-linearity can be combined with a flexible visual shape language to conform content-wise narration to visual expressiveness in real-time.
\end{abstract}

\section{Motivation}

\subsection{Graphic Styles and Visual Narratives}

Visual design professionals use specific styles and composition principles to emphasize the content of a narrative and convey emotions to the viewer implicitly. The term "visual communication", which is used a lot in design industries such as the advertising business, implies that graphic design has not only aesthetic purposes but can be used specifically to deliver messages between two parties. In each case an attempt is made to reinforce the content of the message to be transported, for example by the additional application of psychological perception, and to guide the attention of the target group towards a specific objective. In [1] we already discussed such visual communication as used for in-game landscape design as well as character design for example. 


\subsection{Adapting Interaction in Visual Design}

Static visual media (painting, photography, film) are subject to a fixed dramaturgy. In consequence both the editor who compiles the contents of the narrative and the designer who builds visual footage must decide on a specific dramaturgy at a given point in time. In interactive media, however, the dramaturgy can change constantly and unpredictably according to the user's behavior. In order to respond to the changing dramaturgy in real-time, wouldn't it be more consistent to visually communicate the content-wise changes through dynamic visual design? There already exists some research on this topic: frequently, communication channels such as lighting and camera motion were taken into consideration, notably as they can be flexibly changed at runtime due to their dynamic and technology-related parameters. Any visual element portraying changes in the dramatic emotion at runtime shall hereinafter be referred to as "Adaptive Art".

Additional design features beyond the aforementioned camera and lighting effects may be experimented with, and we focus on shape language in particular. Its use is most evident in sculpture, where it takes on the role of the visual stroke and where it significantly characterizes, in interplay with light and shade, the visible contours and shape surfaces. The connection to the process of virtually modeling wire mesh models in three dimensions is obvious. Distinctive shapes can be used in several occasions. They are found not only in the content manifestation of bodies in space, but also for example in the frame composition as such which is a key principle of filmmaking as described by van Sijll [2] and others. Some research was done by Seif and Zupko [3,4] and by Olivier, Ha and Christie [5] on how different aspects of that area could be applied to gaming and interactive media. While they focus a lot on light and camera work, we present a scenario in which, at runtime, the shapes of objects adapt to dramaturgical values. A similar understanding of the meaning of visual shape was described by Halper et al. [6], for example.

\section{Technical Interpretation}

To implement our adaptive objects we store various mesh deformations using Shape Keys. In contrast to the facial animation our deformations intend to depict the dramatic "look and feel" more than change in content-wise meaning. In addition we use the Shape Keys not for obviously visible animations, but for soft and progressive deformations at the time of the change in the emotional mood of the scene.

Fig. 1 shows an example of the use of the Shape Key mechanism for implementation of a mood-dependent shape language. Therefore the model was initially shaped to have a positive emotional style (left) so that the tree appears in edgy shapes, but the overall shape draws a corpulent body to show vitality. In a second Shape Key (right image side) a contrary mood was created. Using the Shape Key mechanism it is not only possible to visualize two extreme moods, but also to realize flexible transitions. Dramatically this means in case of 


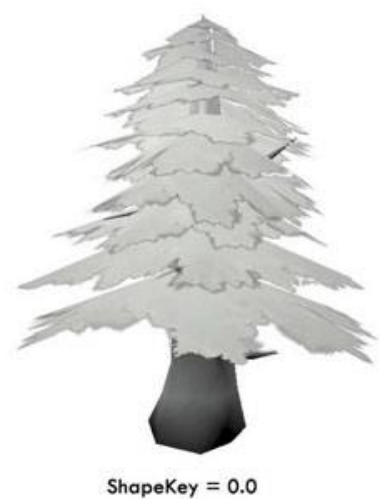

ShapeKey $=0.0$

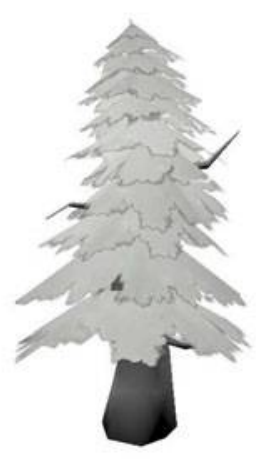

ShapeKey $=0.5$

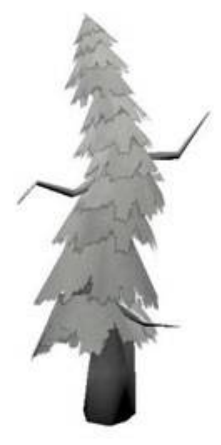

ShapeKey $=1.0$

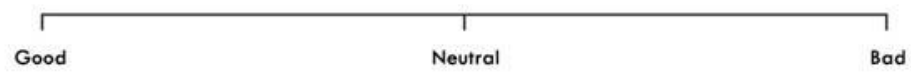

Fig. 1. Dramaturgically adjustable object at three shape-deformation steps

a simple positive-negative scale that the object shows a kind of neutral state in the middle of the blending range. If the dramaturgy changes towards the better or the worse mood, also the Shape Key factor is adjusted in one or the other direction. In this way the creative transformation is mapped to numeric values that can be then easily bound to data sources like an emotion model.

\section{Reflection and Further Perspective}

From feedback of players going through a test scenario we expect to get knowlegde about how far the efforts of creating adaptive image material are worth being made compared to static elements. It is conceivable that for atmospherically dense scenarios as they occur in interactive storytelling, additional channels like coloring and image editing effects are necessary to make the dramaturgy visually "perceptible" and to satisfy the high visual quality today's interactive entertainment customers are used to see, as described recently by Seif El-Nasr[7] for example. If graphical adaption succeeds, it may help to engage these users deeply emotional. Although some computer games that deal with moral decisions like "Black and White", "Star Wars" or "Fable" implement visual reflection of content-wise emotion and mood already, they often use a limited set of techniques like just changing texture colors. Reshaping objects at run-time as introduced in this paper, may help to extend the supply of visual feedback methods.

An ambiguous characteristic of our approach is the danger of substantive change. When an object deforms its content, its meaning also changes inevitably which in turn is the basis for the emotional assessment. Too extreme changes therefore might change the narrative meaning so much that potentially unwanted overlaps with the narrative content may happen. 
Acknowledgments. Parts of the work presented in this paper are co-funded by the German Academic Exchange (DAAD) within the RISE (Research Internships in Science and Engineering) programme http://www.daad.de/rise.

\section{References}

1. Bühling, R., Wißner, M., André, E.: Visual Communication in Interactive Multimedia. In: Dickmann, L., Volkmann, G., Malaka, R., Boll, S., Krüger, A., Olivier, P. (eds.) SG 2011. LNCS, vol. 6815, pp. 159-162. Springer, Heidelberg (2011)

2. van Sijll, J.: Cinematic storytelling: The 100 most powerful film conventions every filmmaker must know. Michael Wiese Productions, Studio City (2005)

3. El-Nasr, M.S., Zupko, J., Miron, K.: Intelligent lighting for a better gaming experience. In: CHI 2005 Extended Abstracts on Human Factors in Computing Systems, CHI EA 2005, pp. 1140-1141. ACM, New York (2005)

4. Zupko, J., El-Nasr, M.S.: System for automated interactive lighting (sail). In: Proceedings of the 4th International Conference on Foundations of Digital Games, FDG 2009, pp. 223-230. ACM, New York (2009)

5. Olivier, P., Ha, H.N., Christie, M.: Smart approaches to lighting design. Information Technology 50, 149-156 (2009)

6. Halper, N., Mellin, M., Herrmann, C.S., Linneweber, V., Strothotte, T.: Psychology and Non-Photorealistic Rendering: The Beginning of a Beautiful Relationship. In: Mensch \& Computer 2003: Interaktion in Bewegung, pp. 277-286. G. Teubner (2003)

7. El-Nasr, M.S., Anders Drachen, J.M.: A Scientific Look at the Design of Aesthetically and Emotionally Engaging Interactive Entertainment Experiences. In: Affective Computing and Interaction: Psychological, Cognitive and Neuroscientific Perspectives. Information Science Publishing (2001) 